\title{
Wind Turbine Noise Reduction from Seismological Data
}

Janis Heuel ${ }^{1}$ (janis.heuel@rub.de), Wolfgang Friederich ${ }^{1}$

${ }^{1}$ Institute of Geology, Mineralogy and Geophysics, Ruhr-University Bochum

\section{Introduction}

- Installation of Wind Turbines (WTs) close to a seismologica station leads to worsening in station qualtity (Fig. 1)

-WT noise and earthquake signals have overlapping frequency bands, thus spectral filtering suppresses both signal and noise

- We applied nonlinear thresholding (Fig. 3c; Langston \& Mousavi, 2019) and used a denoising autoencoder (DAE, Fig. 2) with two different time-frequency transformations to separate noise and signal

- DAE learns a sparse representation of the input data and produces two individual masks for signal and noise (Fig. 2c)

- To train the DAE, we used high signal to noise ratio events and added natural noise from a seismological station surrounded by WTs

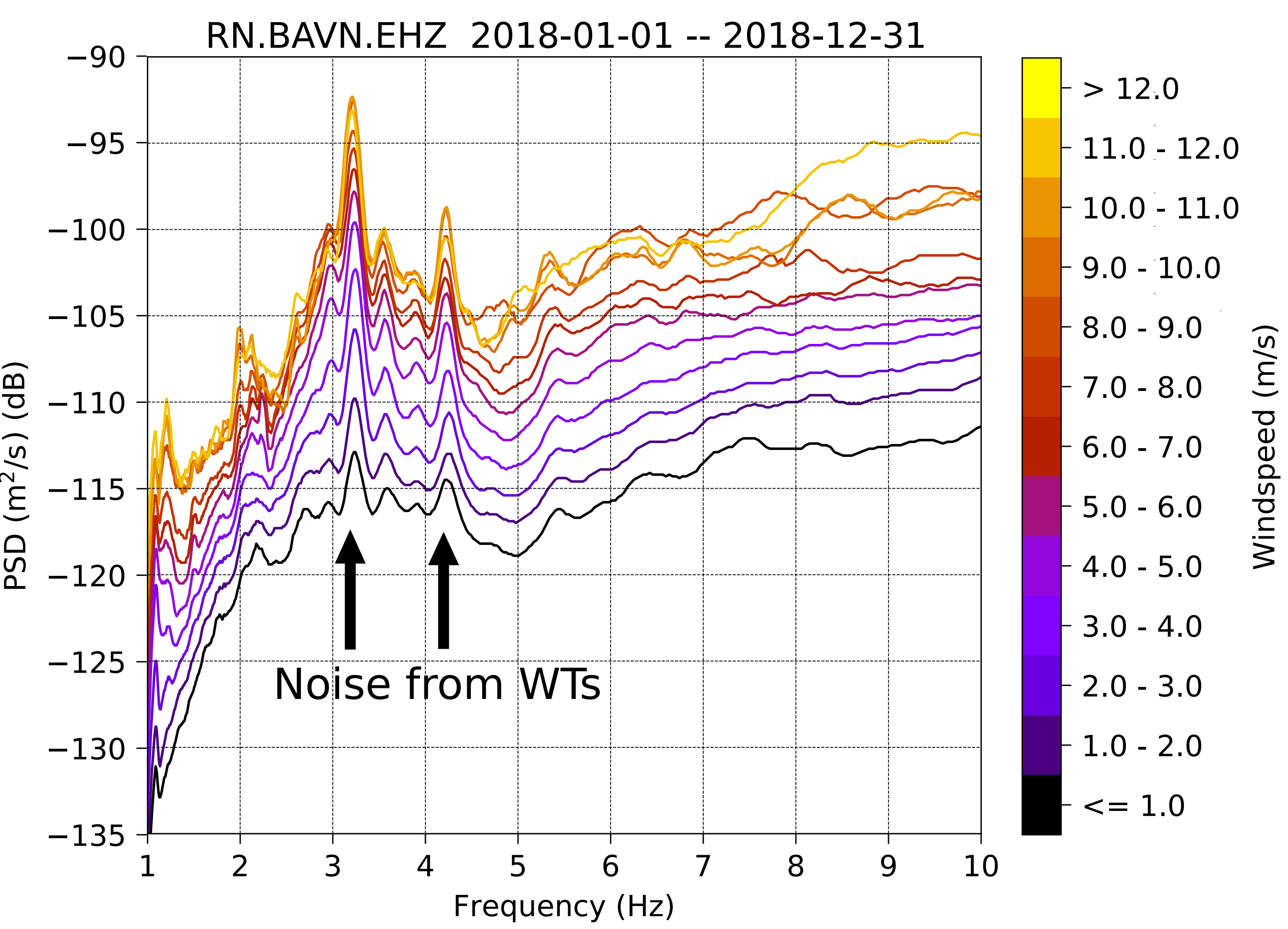

Fig 1: Correlation of power spectral density curves and hourly windspeed measurements for the vertical channel of station BAVN. Arrows indicate frequency peaks caused by surrounding wind turbines.
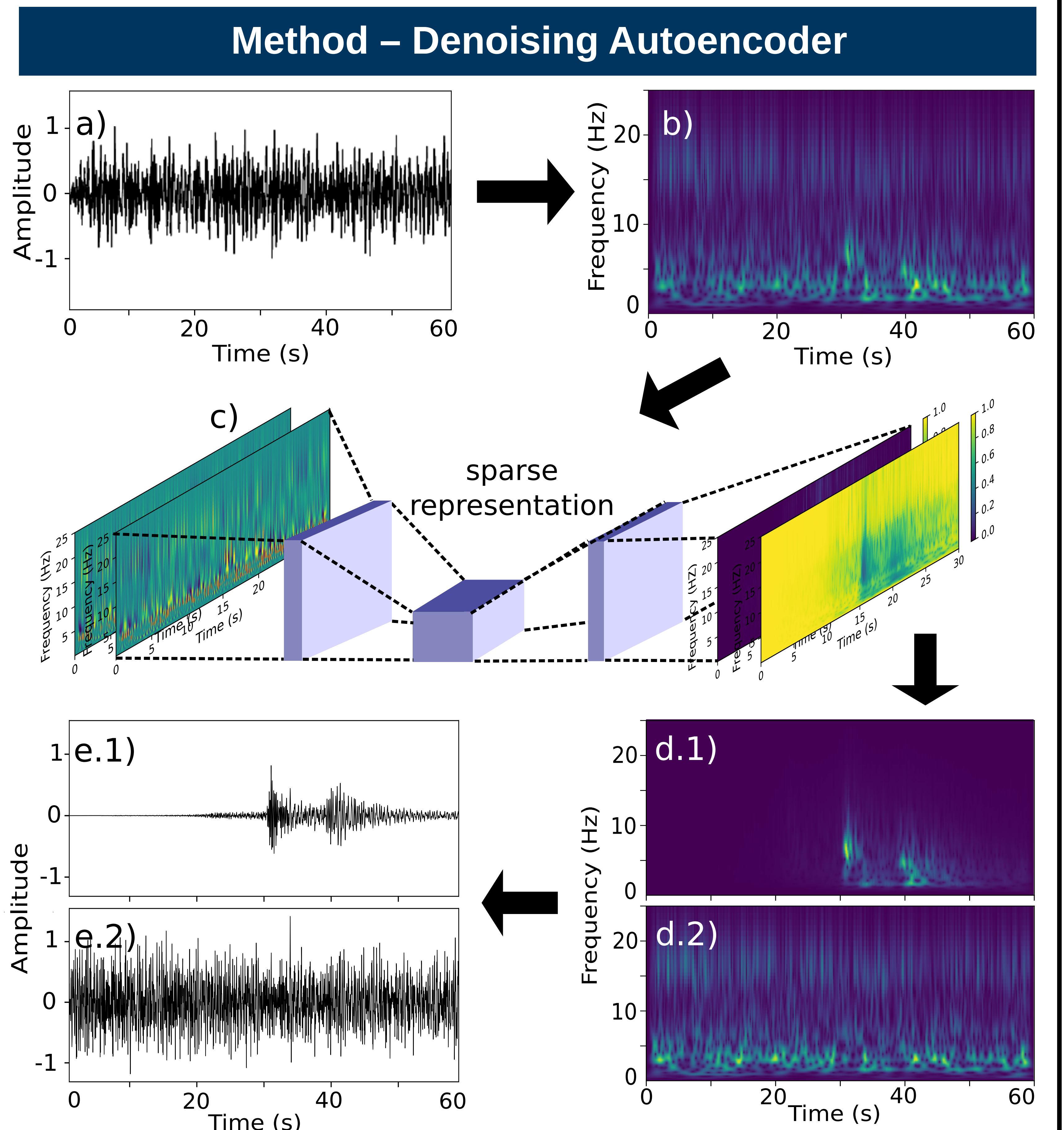

Fig. 2: Data flow of denoising (adapted from Zhu et al., 2019). First, the input data (a) are transformed into time-frequncy (TF) domain (b) either by short-time Fourier transform (STFT) or continous wavelet transform (CWT). The real and imaginary part are fed into a denoising autoencoder (DAE) which produces two masks for signal and noise (c). The masking functions are applied to the noisy TF coefficients to get TF coefficients for signal (d.1) and noise (d.2). Finally, both modified TF coefficients are transformed back into time domain to get signal (e.1) and noise (e.2).

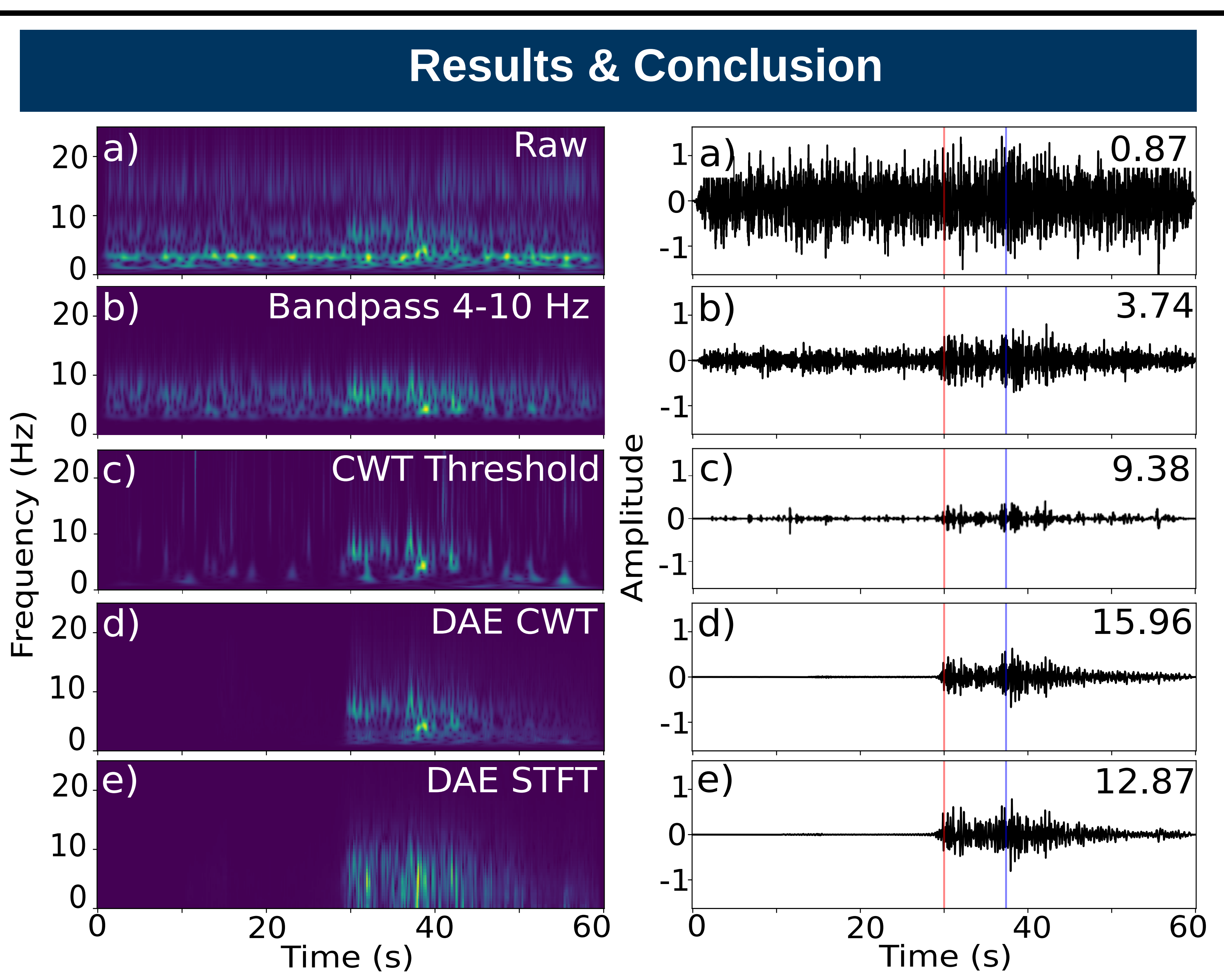

Fig. 3: Comparison of denoising techniques on real data (vertical component). Red and blue lines represent $\mathrm{P}$ - and S-arrival estimated from onsets at other stations. Numbers in waveform plots are the signal to noise ratio. DAE with CWT as time-frequency transform performs best.

- Threshold function results in spiky recovered signal

- DAE separates signal and noise, even if both share a common frequency band

- DAE is limited to recover signals larger than input noisy data because mask functions have values between $[0,1]$

\section{References}

angston, C. A., \& Mousavi, S. M. (2019). Separating Signal from Noise and from Other Sangston, C. A., \& Mousavi, S. M. (2019). Separating Signal from Noise and from Oth
Tignal Using Nonlinear Thresholding and Scale-Time Windowing of Continuous Wav
Transforms. Bulletin of the Seismological Society of America, 109(5), 1691-1700. Zhu, W., Mousavi, S. M., \& Beroza, G. C. (2019). Seismic signal denoising and
decomposition using deep neural networks. IEEE Transactions on Geoscience and 